\title{
Temperature dependence of the electrical properties of the carbon nanotube/polymer composites
}

\author{
Q. Li $^{1,2}$, Q. Z. Xue ${ }^{1 *}$, X. L. Gao ${ }^{1}$, Q. B. Zheng 1 \\ ${ }^{1}$ College of Physics Science and Technology, China University of Petroleum, Dongying, Shandong 257061, \\ People's Republic of China \\ 2Department of Materials and Chemical Engineering, Taishan University, Tai'an, Shandong 271021, \\ People's Republic of China
}

Received 17 July 2009; accepted in revised form 4 September 2009

\begin{abstract}
In this paper, pristine and oxidized multi-walled carbon nanotube (MWCNT)/poly(vinylidene fluoride) (PVDF) composites were prepared and the temperature dependence of some electrical properties of these composites were studied. It is found that the transition temperature $\left(T_{t}\right)$, from positive temperature coefficient (PTC) to negative temperature coefficient (NTC) effect, of the oxidized MWCNT/PVDF composites shifted to a higher temperature. The shift of the $T_{t}$ of the oxidized MWCNT/PVDF composites can be attributed to the chemical functionalization of the MWCNTs. The dielectric constants of these composites are enhanced remarkably, which can be understood by the interfacial polarization effect. The largest dielectric constant of 3600 is obtained in the composite with about 8 vol\% oxidized MWCNTs at $1 \mathrm{kHz}$. The dielectric constants of these composites increase firstly and then decrease with increasing temperature. However, when the temperature reaches a higher value, the dielectric constants increase again with increasing temperature. The 'wave' phenomenon of the temperature dependence of the dielectric constants can be understood by the temperature dependence of the interfacial polarization.
\end{abstract}

Keywords: polymer composites, carbon nanotube, electrical properties, thermal properties

\section{Introduction}

It is well known that the resistance of the carbon black (CB) or other conductive particle filled semicrystalline polymer composites exhibits a sharp increase when the temperature reaches the melting temperature $\left(T_{m}\right)$ of the polymers. This phenomenon is referred as the positive temperature coefficient (PTC) effect. When the temperature is above $T_{m}$, the resistance of the composites will decrease. This is the negative temperature coefficient (NTC) effect. The PTC effect and NTC effect of the CBfilled semi-crystalline polymer composites have been investigated for a long time. In recent years, the relative factors of the PTC and NTC effects of CB-filled semi-crystalline polymer composites have been studied in detail [1-4]. The results showed that both the molecular weight of the polymer matrix and the size of $\mathrm{CB}$ could influence the percolation threshold and the PTC temperature, at which a sharp increase of the resistance of these composites occurs. Zhang et al. [4] studied the electrical properties of the CB-filled ultra-high molecular weight polyethylene (UHMWPE). They found that the percolation threshold of these composites decreases with the increase of the molecular weight of UHMWPE, and with the decrease of the particle size of CB. The PTC temperature of the CB-filled UHMWPE composite with the UHMWPE molecular weight of 145 millions decreases with the increase of CB size. The PTC composites have 
many important applications, such as the over-current protectors and sensors. However, CB-filled polymer composites require a high $\mathrm{CB}$ concentration. This means that the excellent properties of the polymer matrix will be influenced.

Since the discovery of carbon nanotubes (CNTs) in 1991, they have been widely studied in several fields because of their extraordinary properties [5]. $\mathrm{CNT} /$ polymer composite is one of the important applications of the CNTs $[6,7]$. The properties of the $\mathrm{CNT} /$ polymer composites will be enhanced remarkably with small CNT concentration. The application of the CNT/polymer composites under different temperatures is the problem that we must think about. He et al. [8] studied the temperature dependence of electrical properties of the multiwalled carbon nanotube (MWCNT) filled high density polyethylene (HDPE) composites, and found an obvious PTC effect and a less obvious NTC effect in these composites with a low MWCNT concentration. The PTC intensity of the MWCNT/ HDPE composite with $5.4 \mathrm{wt} \%$ MWCNTs reached $10^{4}$. After $\gamma$-ray irradiation, the NTC effect of these composites could be further eliminated. Poly(vinylidene fluoride) (PVDF) and its copolymers, as semicrystalline polymer materials, have attracted more attention for their broad applications in electromechanical systems $[9,10]$. The mechanical properties of the PVDF polymer are better than those of the polyethylene (PE), which is the traditional PTC polymer. And the $T_{m}$ of PVDF is also higher than that of PE, which means that the application range is wider than that of PE polymer.

In this paper, we investigated the temperature dependence of electrical properties of the pristine and oxidized MWCNT/PVDF composites. Because of the high aspect ratio, the percolation threshold is much smaller than that of CB filled polymer composites. The dielectric constant of these composites is enhanced remarkably. The temperature dependence of electrical properties of these composites showed that the transition temperature $\left(T_{t}\right)$, from PTC effect to NTC effect, of the oxidized MWCNT/ PVDF composites shifted to a higher temperature. The dielectric constants of these composites increase with increasing temperature, and then decrease. However, the dielectric constants increase again when the temperature reaches a higher temperature. The temperature dependence of the dielectric constants indicated a 'wave' phenomenon.

\section{Experimental section}

\subsection{Materials}

The MWCNTs (purity > 95 wt $\%$, diameter 10 $20 \mathrm{~nm}$, length about $30 \mu \mathrm{m}$, density $2.1 \mathrm{~g} / \mathrm{cm}^{3}$ ) synthesized by Chemical Vapor Deposition were purchased from Chengdu Organic Chemistry Co. Ltd., Chinese Academy of Science. The PVDF powder (particle size about $50 \mu \mathrm{m}$, density $1.7 \mathrm{~g} / \mathrm{cm}^{3}$ ) was purchased from 3F Co. Ltd., Shanghai, China. The ethanol (AR), concentrated nitric acid (AR), and concentrated sulfuric acid (AR) were purchased from Tianjin Chemical Co. Ltd., China. Ultrasonicator (HK2200, 100W) was purchased from High Kugod Science Instrument Co. Ltd., Shanghai, China. LCR digital meter (TH2828) was purchased from Tonghui Electronic Co. Ltd., Changzhou, China. Deionized water was supplied by the University of Petroleum, China.

\subsection{Sample preparation}

Briefly, $1 \mathrm{~g}$ of the pristine MWCNTs was suspended in $100 \mathrm{ml}$ mixture of concentrated sulfuric acid and concentrated nitric acid $(3: 1[\mathrm{v} / \mathrm{v}])$ for $6 \mathrm{~h}$ with the ultrasonicator. The MWCNTs were filtrated and washed with deionized water until $\mathrm{pH} 5-7$, and then dried at $55^{\circ} \mathrm{C}$ in a vacuum for $8 \mathrm{~h}$ $[11,12]$. The oxidized MWCNTs were obtained. In order to form a stable suspension, the pristine and oxidized MWCNTs were ultrasonically dispersed in ethanol for $3 \mathrm{~h}$ respectively. Then the PVDF powder was poured into this suspension. The PVDF powder was swollen by ethanol and the mixture was ultrasonically dispersed for another $2 \mathrm{~h}$. The mixture was dried for $3 \mathrm{~h}$ at $70^{\circ} \mathrm{C}$ to remove any excess ethanol. The agglomerates of these composites were broken into powder. The composite powder was cold pressed to disk-shape sample with $15 \mathrm{~mm}$ in diameter and $2 \mathrm{~mm}$ in thickness at $20 \mathrm{MPa}$. Then the samples were kept at $200^{\circ} \mathrm{C}$ for $3 \mathrm{~h}[13,14]$.

\subsection{Measurements}

All samples were polished to control their flatness, and silver paste was painted on both sides of the samples to get an ohmic contact with the electrodes. The alternating current (ac) electrical properties of the samples were measured by using two-probe method with LCR digital meter in the 


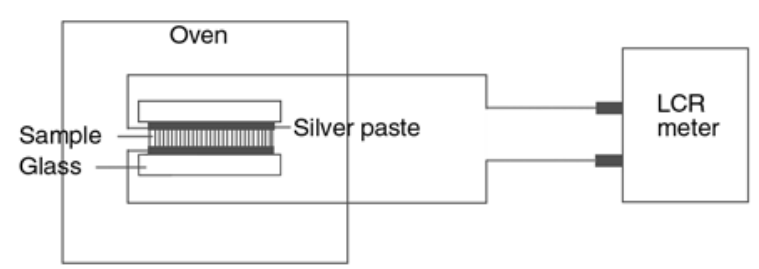

Figure 1. The scheme of the measurement of the electrical properties at different temperatures

frequency range of $20-10^{6} \mathrm{~Hz}$ at room temperature. The temperature dependence of the electrical properties was measured by putting the samples in an oven [4]. Figure 1 illustrates the measurement system.

\section{Results and discussion}

\subsection{Characterization of CNTs and composites}

Figure 2 shows the transmission electron microscopy (TEM) images of the pristine and oxidized MWCNTs and the scanning electron microscope (SEM) images of the pristine and oxidized MWCNT/PVDF composites in our experiment. As shown in Figures $2 \mathrm{a}$ and $2 \mathrm{~b}$, the impurity, for

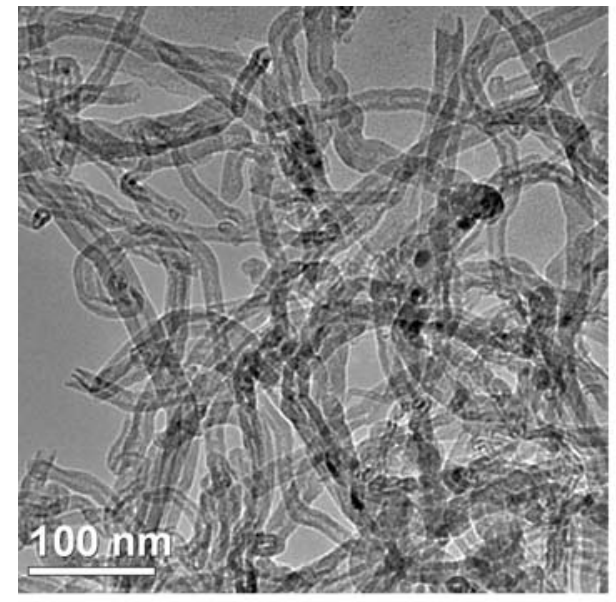

a)

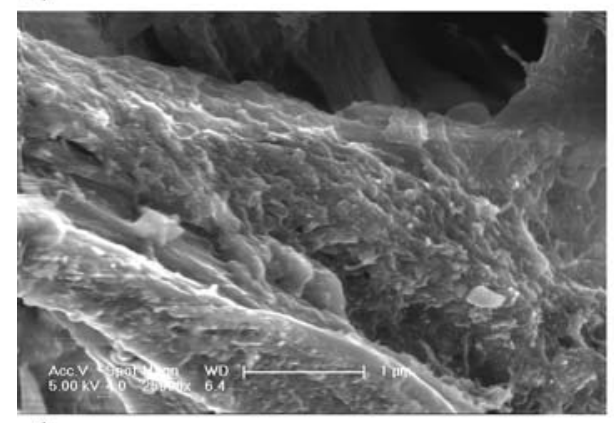

c) instance the amount of amorphous carbon materials and catalysts, in oxidized MWCNTs is less than that in pristine MWCNTs. The MWCNTs were purified. The length of the MWCNTs became shorter by the chemical functionalization. Figures $2 \mathrm{c}$ and $2 \mathrm{~d}$ show the SEM images of the composite sections, which indicate that the MWCNTs were dispersed in the PVDF matrix homogeneously.

\subsection{Electrical properties of these composites}

Figure 3 shows the electrical conductivity and the dielectric constants of the pristine and oxidized MWCNT/PVDF composites as a function of the volume fraction of the MWCNTs measured at room temperature and $1 \mathrm{kHz}$. The electrical conductivity of these two kinds of composites increased significantly with low MWCNT concentration. The conductivity can be analyzed with the critical MWCNT concentration, percolation threshold $p_{c}$, by the following scaling law [14-16] (Equation (1)):

$$
\sigma_{e f f} \propto \sigma_{i}\left(p_{c}-p_{\mathrm{MWCNT}}\right)^{-s^{\prime}}, \text { for } p_{c}>p_{\mathrm{MWCNT}}
$$

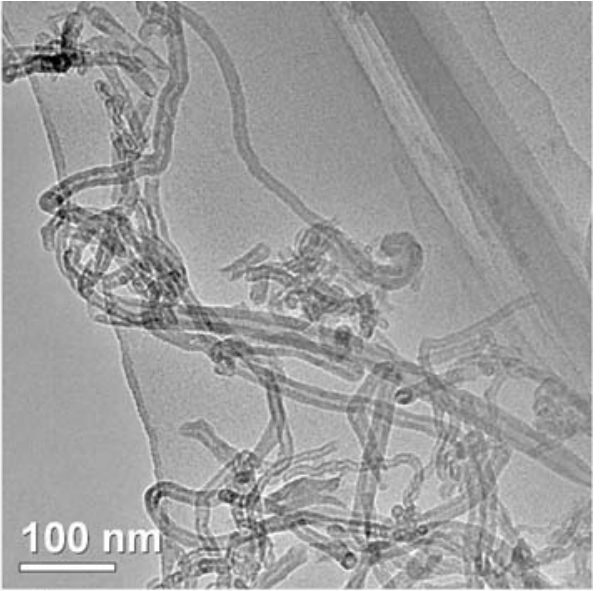

b)

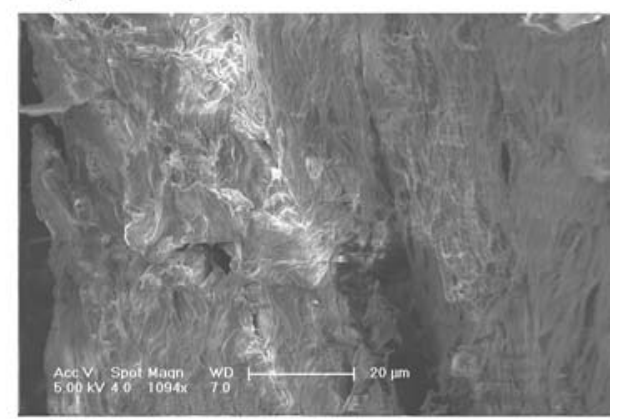

d)

Figure 2. TEM images of a) pristine; and b) oxidized MWCNTs; SEM images of c) pristine, and d) oxidized MWCNT/PVDF composite sections 


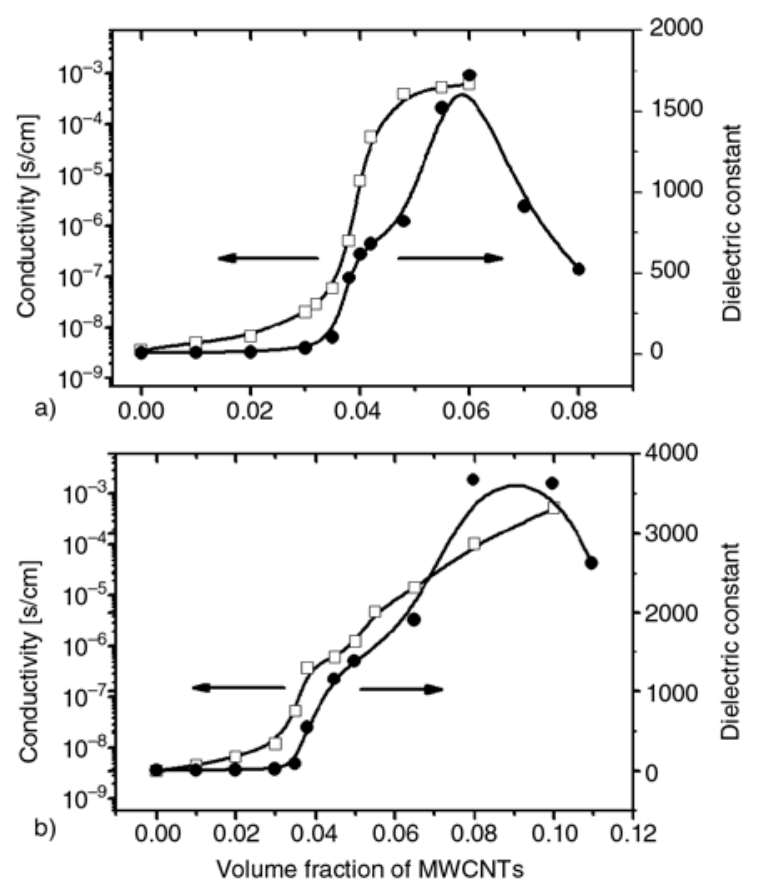

Figure 3. Dependence of the electrical conductivity and dielectric constant of a) pristine MWCNT/PVDF composites; and b) oxidized MWCNT/PVDF composites on the MWCNT concentration

The percolation threshold of these composites by calculating is about $3.8 \mathrm{vol} \%$. The conductivity of the pristine MWCNT/PVDF composites increases remarkably near the percolation threshold, and exhibits a typical insulator-conductor transition. However, the conductivity of the chemically functionalized MWCNT/PVDF composites increases slowly with increasing the MWCNT volume fraction. The reason for the electrical properties of these composites can be attributed to the tunneling conduction mechanism and percolation phenomenon. The carboxylic groups on the surface of MWCNTs can decrease the tunneling currents. In other words, it becomes difficult for the tunneling event to occur, if there are carboxylic groups between MWCNTs. Therefore, the tunneling current will decrease relatively, and the conductivity will increase slightly with increasing MWCNT concentration [14-16].

The dielectric constant increases remarkably when the concentration of MWCNTs approaches to about 6 and $8 \mathrm{vol} \%$ for the pristine and oxidized MWCNT/PVDF composites, respectively, and then the dielectric constants decrease with increasing MWCNTs concentration. As we noted, the largest dielectric constants of these two kinds of composites are different. The largest dielectric constant of the pristine MWCNT/PVDF composites is 1700, with about 6 vol\% MWCNTs at $1 \mathrm{kHz}$. However, it reaches 3600 for the oxidized MWCNT/PVDF composite with about 8 vol\% oxidized MWCNTs. The dielectric properties of the composites can be understood by the percolation theory and interfacial polarization effect. In these composites, the MWCNTs were distributed in the insulting matrix, which can form a lot of interfaces. For these composites, a thin insulating layer of PVDF was combined with the MWCNTs to form the nanoscale structure in these composites. The large $\Pi$-orbital of the MWCNTs could provide large domains for nomadic electrons. These electrons can be orientated under electric field, and interfacial polarization can take place [15]. As the MWCNT concentration increases, the number of the interfaces increases. So dielectric constant increases with increasing MWCNT concentration. When the concentration of MWCNTs is larger than the percolation threshold of the composites, some MWCNTs will connect with each other, and even agglomerated again. The interfaces between the MWCNTs and the PVDF will decrease. The dielectric constant will decrease with further increasing MWCNT concentration.

The different largest dielectric constant of these two kinds of composites can be attributed to the chemical functionalization. Compared with the pristine MWCNTs, the length of the MWCNTs became shorter by the chemical functionalization, so that there will need more MWCNTs to form the conductive nets. So the interfaces formed between the oxidized MWCNTs and PVDF matrix will be larger than that between pristine MWCNTs and PVDF matrix. Moreover the carboxylic groups are the electron-withdrawing groups, and this will strengthen interfacial polarization. Therefore, the different largest dielectric constants of MWCNT/ PVDF composites can be attributed to the different MWCNT concentration and the chemical functionalization [16].

Furthermore, the measured dielectric constant values show a divergence at the percolation threshold. The reasons of these changes of dielectric constants in the neighborhood of the percolation threshold may be caused by this factor. As shown in Figure 3, the percolation transition region is wide, the variation of dielectric constants will occur in the percolation transition region, and the percolation threshold calculated by Equation (1) is only the 
beginning value of the percolation transition scope. This caused the divergence $[15,16]$.

\subsection{Temperature dependence of the impedance}

Figure 4 shows the temperature dependence of impedance of these two kinds of MWCNT/PVDF composites. As shown in this figure, the PTC and NTC effects of these composites are both small. The impedance of the composites at $T_{t}$ is only 2-4 times larger than that at room temperature.

It has been accepted that the PTC effect is due to the different thermal expansion coefficients of the polymer matrix and the filler [1-4]. At lower temperature the conductive fillers imbedded in the insulator can connect with each other, which form the conducting paths. When the temperature is lower than $T_{t}$, the expansion of the polymer matrix will separate the fillers that connected with each other before, and the impedance of the composites increases with increasing temperature. When the temperature is higher than $T_{t}$, the PVDF crystalline
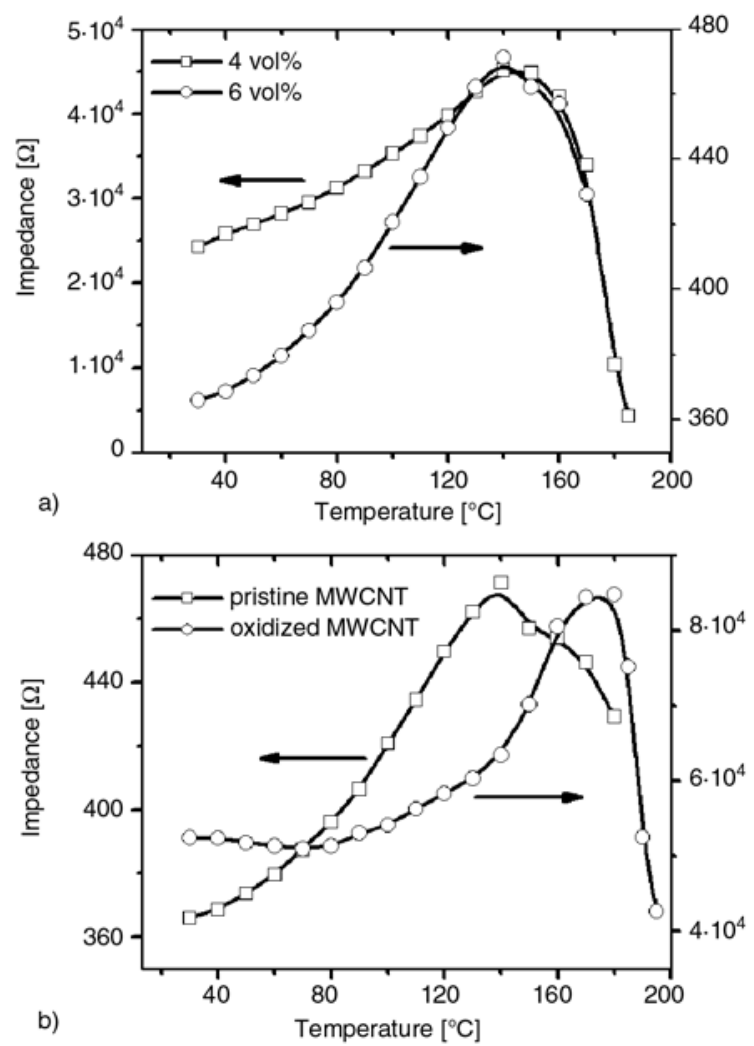

Figure 4. Temperature dependence of the impedance for these two kinds of MWCNT/PVDF composites; a) 4 and 6 vol\% pristine MWCNT/PVDF composites, b) 6 vol $\%$ pristine and oxidized MWCNT/PVDF composites phase begins to melt, transforming from a semicrystalline phase to the rubbery flow region. Therefore, the fluidity of the polymer matrix and the mobility of the MWCNTs will be enhanced in rubbery flow region. This will make it easier for the fillers to connect with each other than before so that the impedance decreases with a further increasing temperature. On the other hand, the electrons will capture more energy when the temperature increases, and they can overcome the potential barrier easily. So the impedance decreases with increasing temperature. The small temperature coefficients of the MWCNT/PVDF composites can be attributed to the one-dimension structure of CNTs and the conducting network formed by the MWCNTs in composites. At low temperature, the MWCNTs contacted each other in the PVDF matrix. Because of the one-dimension structure of the CNTs, the contact probability of one CNT and anthor CNT is larger than that between two CB particles. Compared with the CB network, CNT network is very difficult to disconnect because of the connection with each other. This reason caused the small PTC effect of these composites.

As shown in Figure $4 b$, the impedance-temperature curves show that the $T_{t}$ of the oxidized MWCNT/ PVDF composites shifts to a higher temperature. However, as shown in Figure 5, the DSC curves show that the $T_{m}$ of these two kinds of composite powders is approximately equal. The $T_{m}$ of the pure PVDF is slightly higher than the composites. MWCNTs can be considered as the nucleation agent and improve the crystallinity of the polymer matrix. The defects of the polymer matrix crystal

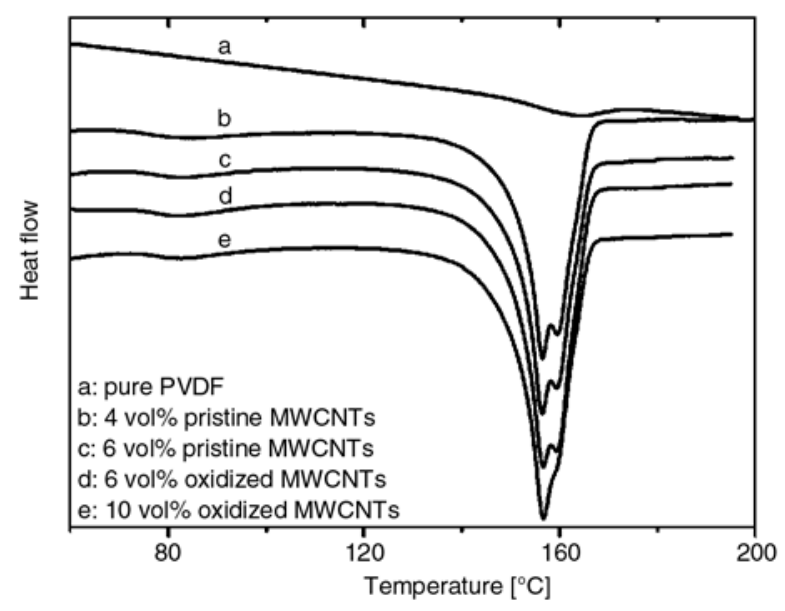

Figure 5. DSC curve of the pure PVDF and the MWCNT/PVDF composites with various MWCNT concentration 
may be introduced by the MWCNTs, and the lamellae thickness may be slightly reduced. So the $T_{m}$ of the composites was decreased slightly [17]. According to the polymer theory, the $T_{t}$ should be approximately equal to $T_{m}$ of the polymer. We think that there are two reasons for this difference. Firstly, the interface effect between the MWCNTs and the PVDF matrix [18]. The carboxylic acid groups were introduced to the surface of the MWCNTs in the oxidized MWCNT/PVDF composites. The thermal interfacial resistance between MWCNTs and polymer matrix can be reduced. The thermal conductance property of oxidize MWCNT/ PVDF composites is better than that of pristine MWCNT/PVDF composites. So this will consume more thermal energy to melt polymer matrix [18-21]. Secondly, the carboxylic groups on the surface of the MWCNTs can be imbedded into the polymer matrix, which cause the oxidized MWCNT/ PVDF composites to be more compact. So the $T_{t}$ of the oxidized MWCNT/PVDF composites shifts to a higher temperature. In a word, the interface effect between the oxidized MWCNTs and the polymer matrix may be the major reason for the change of the $T_{t}$.

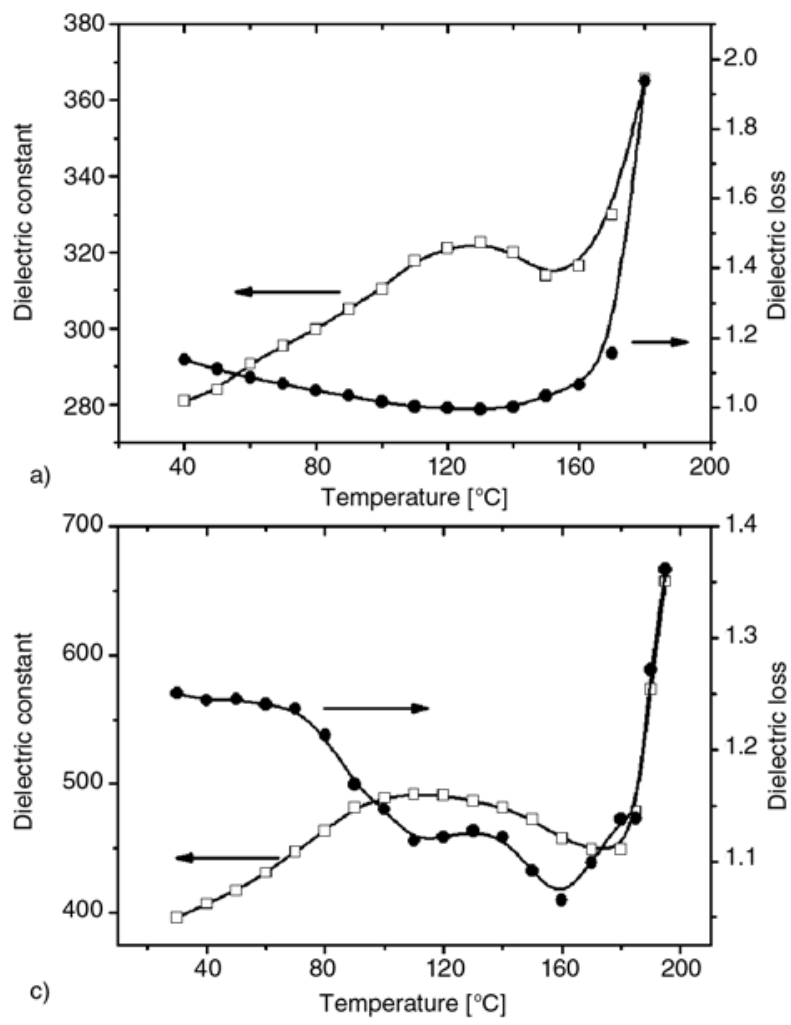

\subsection{Temperature dependence of dielectric properties}

The temperature dependence of the dielectric properties of the pristine and oxidized MWCNT/PVDF composites are shown in Figure 6. The dielectric constants increase firstly, and then decrease with increasing temperature. After that, the dielectric constants increase again with increasing temperature when the temperature reaches a higher temperature. The dielectric loss decrease first, and then increase with increasing temperature. All the specimens have the similar properties. In order to find out the mechanism of the temperature dependence of the dielectric properties, we investigated the temperature dependences of the impedance and dielectric properties of the pure PVDF matrix. Figure 7 shows the temperature dependences of the impedance and dielectric properties of the pure PVDF matrix at $1 \mathrm{kHz}$. As shown in Figure 7, the temperature dependence of the dielectric constants of the PVDF matrix shows the same 'wave' phenomenon.

Electric modulus formalism is a good method to study the polarization effect. The electric modulus can be calculated by the Equation (2) [22, 23]:

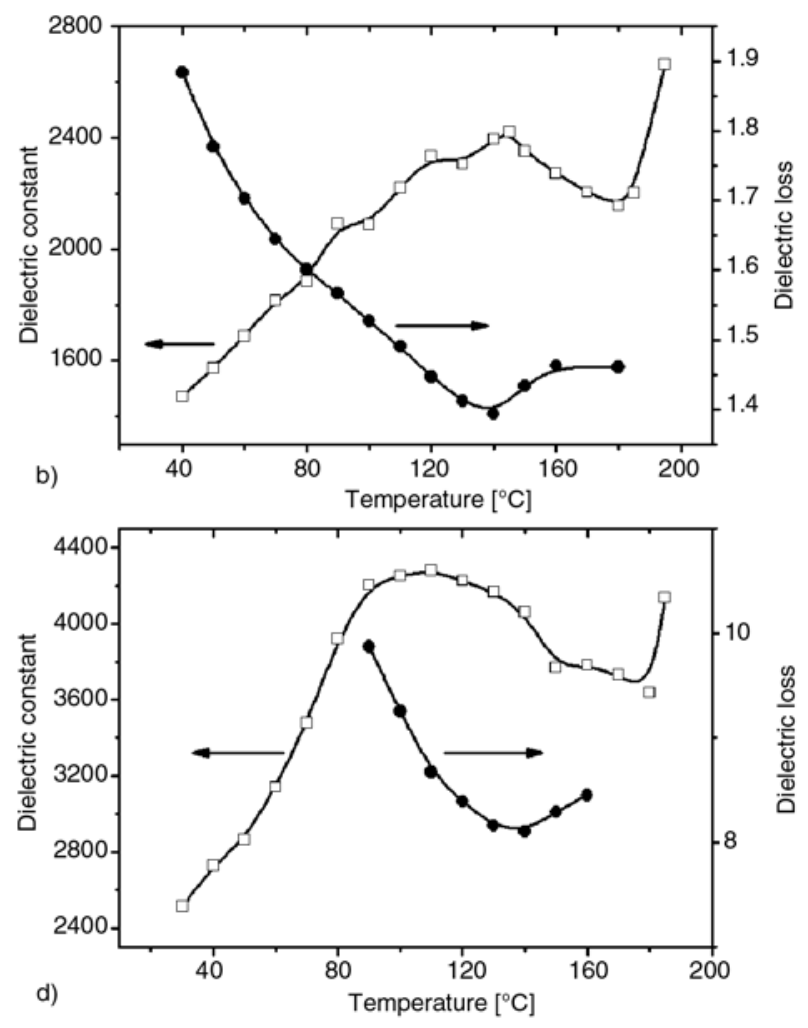

Figure 6. Temperature dependence of the dielectric constants and dielectric loss of a) 4 vol\%, b) 6 vol\% pristine MWCNT/PVDF composites; and c) 6 vol\%,d) $10 \mathrm{vol} \%$ oxidized MWCNT/PVDF composites at $1 \mathrm{kHz}$ 


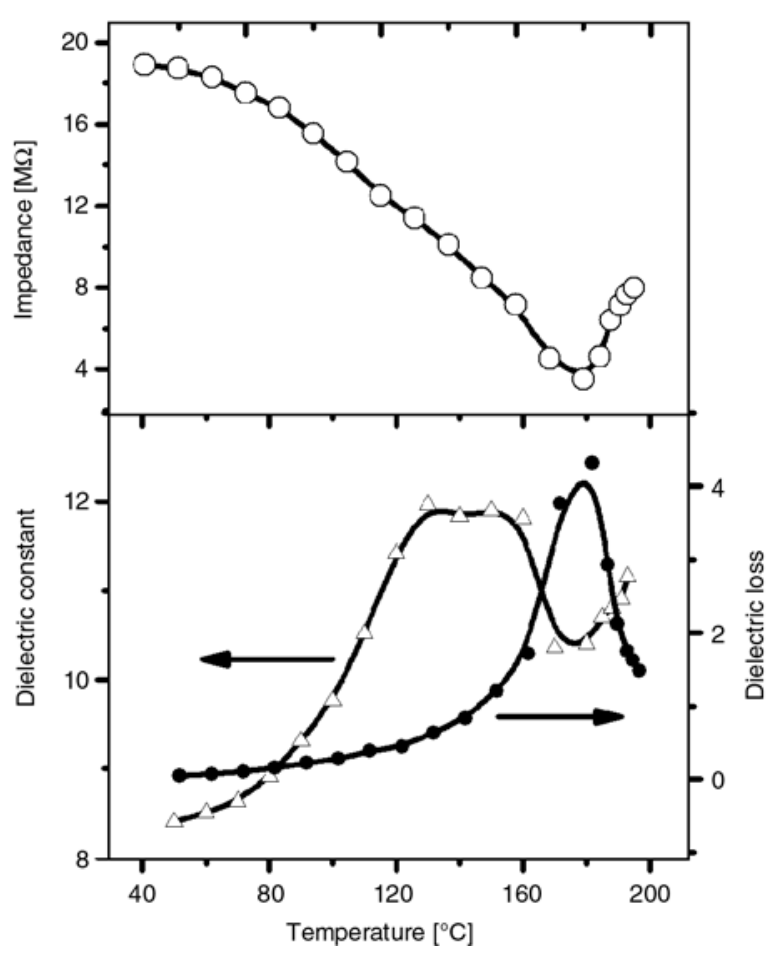

Figure 7. Temperature dependence of the electrical properties of the pure PVDF matrix

$$
\begin{array}{r}
M^{*}=\left(\varepsilon^{*}\right)^{-1}=M^{\prime}+i M^{\prime \prime}= \\
\frac{\varepsilon^{\prime}}{\varepsilon^{\prime 2}+\varepsilon^{\prime \prime 2}}+i \frac{\varepsilon^{\prime \prime}}{\varepsilon^{\prime 2}+\varepsilon^{\prime \prime 2}}
\end{array}
$$

Because of the instrument precision, we do not get the values of the electric modulus of the MWCNT/ PVDF composites at low frequency. The electric modulus formalism of the pure PVDF is calculated. Figure 8 shows the electric modulus formalism of the pure PVDF. As shown in this figure, the values of real components $\left(M^{\prime}\right)$ increase with frequency and reach a constant value as expected. The values of imaginary components $\left(M^{\prime \prime}\right)$ show a peak at low frequency at 110 and $140^{\circ} \mathrm{C}$. Relaxation peaks are attributed to an interfacial polarization process. According to the electric modulus formalism of the pure PVDF matrix, the temperature frequency of dielectric properties of the MWCNT/PVDF composites can be explained as follows [24, 25]. When the temperature is lower than $T_{t}$, the expansion of the polymer matrix will separate the fillers that connected with each other before. The interfaces between the MWCNTs and the polymer matrix will increase, and the dielectric constant will increase. When the temperature is higher than $T_{t}$, the PVDF crystalline phase begins to melt, transforming from

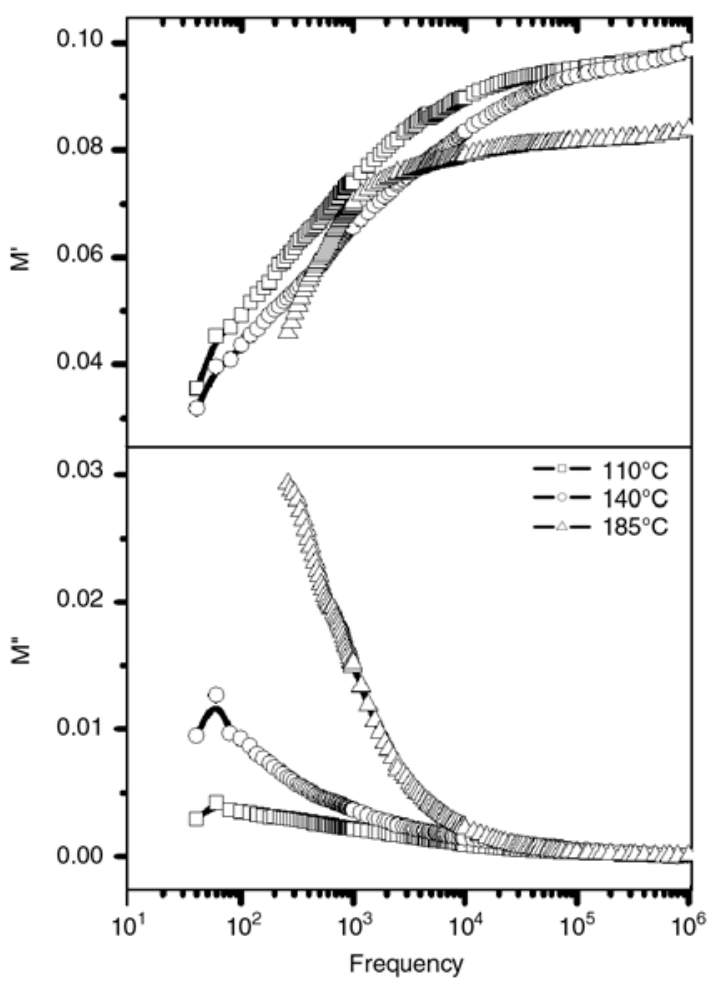

Figure 8. Real $M^{\prime}$ and imaginary $M^{\prime \prime}$ of the electric modulus formalism of the pure PVDF versus frequency at different temperatures

a semi-crystalline phase to the rubbery flow region. This will make it easier for the fillers to connect with each other. The interfaces between the MWCNTs and the polymer matrix will decrease. So the dielectric constant will decrease. However, the nomadic electrons will get larger energy and agglomerated on the interfaces at higher temperatures because the fluorine atom is an electron-withdrawing group. The polarization will be enlarged, even exceed the influence of MWCNTs connect again. The dielectric constant increases again. We do not get the values of the electric modulus of the PVDF composites at higher temperatures (for example $185^{\circ} \mathrm{C}$ ) at low frequency for the reason of instrument precision. So the electrode effect should also be considered for the later increase.

\section{Conclusions}

The pristine and oxidized MWCNT/PVDF composites were fabricated by solution blending. The percolation threshold of these composites is smaller than that of $\mathrm{CB}$ filled polymer composites. The dielectric constant of the composites was enhanced remarkably, which can be understood by interfacial 
polarization effect. The temperature dependence of impedance of these MWCNT/PVDF composites indicates that the PTC and NTC effects of these two kinds of composites are small. Because of the functionalization of the MWCNTs, the transition temperature, from positive temperature coefficient to negative temperature coefficient effect, of the oxidized MWCNT/PVDF composites is larger than that of the pristine MWCNT/PVDF composites. The dielectric constants of these composites increase firstly and then decrease with increasing temperature. However, when the temperature reaches a higher temperature, the dielectric constants increase again with increasing temperature. The 'wave' phenomenon of the temperature dependence of these dielectric constants can be attributed to the interfacial polarization under different temperatures.

\section{Acknowledgements}

This work is supported by 973 National Basic Research Program under Contract No. 2008CB617508, and the Cultivation Fund of the Key Scientific and Technical Innovation Project (No. 708061), Ministry of Education of China, and Program for New Century Excellent Talents in University (NCET-08-0844).

\section{References}

[1] Feng J., Chan C-M.: Double positive temperature coefficient effects of carbon black-filled polymer blends containing two semicrystalline polymers. Polymer, 41, 4559-4565 (2000). DOI: 10.1016/S0032-3861(99)00690-4

[2] Feng J., Chan C-M.: Positive and negative temperature coefficient effects of an alternating copolymer of tetrafluoroethylene-ethylene containing carbon black filled HDPE particles. Polymer, 41, 7279-7282 (2000).

DOI: $10.1016 /$ S0032-3861(00)00095-1

[3] Hindermann-Bischoff M., Ehrburger-Dolle F.: Electrical conductivity of carbon black-polyethylene composites experimental evidence of the change of cluster connectivity in the PTC effect. Carbon, 39, 375-382 (2001).

DOI: $10.1016 / \mathrm{S} 0008-6223(00) 00130-5$

[4] Zhang C., Ma C. A., Wang P., Sumita M.: Temperature dependence of electrical resistivity for carbon black filled ultra-high molecular weight polyethylene composites prepared by hot compaction. Carbon, 43, 2544-2553 (2005).

DOI: $\underline{\text { 10.1016/j.carbon.2005.05.006 }}$
[5] Iijima S.: Helical microtubules of graphitic carbon. Nature, 354, 56-58 (1991). DOI: $\underline{10.1038 / 354056 \mathrm{a} 0}$

[6] Sandler J., Shaffer M. S. P., Prasse T., Bauhofer W., Schulte K., Windle A. H.: Development of a dispersion process for carbon nanotube in an epoxy matrix and the resulting electrical properties. Polymer, 40, 5967-5971 (1999). DOI: 10.1016/S0032-3861(99)00166-4

[7] Barrau S., Demont P., Peigney A., Laurent C., Lacabanne C.: DC and AC conductivity of carbon nanotube-polyepoxy composites. Macromolecules, 36, 5187-5194 (2003). DOI: $10.1021 / \mathrm{ma} 021263 \mathrm{~b}$

[8] He X. J., Du J. H., Ying Z., Cheng H. M., He X. J.: Positive temperature coefficient effect in multiwalled carbon nanotube/high-density polyethylene composites. Applied Physics Letters, 86, 062112/1-062112/3 (2005).

DOI: $\underline{10.1063 / 1.1863452}$

[9] Zhao X-Z., Bharti V., Zhang Q. M., Romotowski T., Tito F., Ting R.: Electromechanical properties of electrostrictive poly(vinylidene fluoride-trifluoroethylene) copolymer. Applied Physics Letters, 73, 2054-2056 (1998).

DOI: $\underline{10.1063 / 1.122365}$

[10] Huang C., Zhang Q. M., Su J.: High-dielectric constant all-polymer percolative composites. Applied Physics Letters, 82, 3502-3504 (2003). DOI: $\underline{10.1063 / 1.1575505}$

[11] Li S., Qin Y., Shi J., Guo Z. X., Li Y., Zhu D.: Electrical properties of soluble carbon nanotube/polymer composite films. Chemistry of Materials, 17, 130-135 (2005).

DOI: $10.1021 / \mathrm{cm} 0491025$

[12] Kim Y. J., Shin T. S., Choi H. D., Kwon J. H., Chung Y-C., Yoon H. G.: Electrical conductivity of chemically modified multiwalled carbon nanotube/epoxy composites. Carbon, 43, 23-30 (2005). DOI: 10.1016/j.carbon.2004.08.015

[13] Owens F. J., Jayakody J. R. P., Greenbaum S. G.: Characterization of single walled carbon nanotube: Polyvinylene difluoride composites. Composites Science and Technology, 66, 1280-1284 (2006). DOI: $10.1016 /$ j.compscitech.2005.10.027

[14] Wang L., Dang Z-M.: Carbon nanotube composites with high dielectric constant at low percolation threshold. Applied Physics Letters, 87, 042903/1-042903/3 (2005).

DOI: $\underline{10.1063 / 1.1996842}$

[15] Dang Z., Wang L., Yin Y., Zhang Q., Lei Q.: Giant dielectric permittivities in functionalized carbonnanotube/electroactive-polymer nanocomposites. Advanced Materials, 19, 852-857 (2007). DOI: $\underline{10.1002 / \mathrm{adma} .200600703}$ 
[16] Li Q., Xue Q., Hao L., Gao X., Zheng Q.: Large dielectric constant of the chemically functionalized carbon nanotube/polymer composites. Composites Science and Technology, 68, 2290-2296 (2008). DOI: 10.1016/j.compscitech.2008.04.019

[17] Zhang S., Zhang N., Huang C., Ren K., Zhang Q.: Microstructure and electromechanical properties of carbon nanotube/poly(vinylidene fluoride trifluoroethylene chlorofluoro ethylene) composites. Advanced Materials, 17, 1897-1901 (2005).

DOI: $10.1002 /$ adma.200500313

[18] Shenogin S., Bodapati A., Xue L., Ozisik R., Keblinski P.: Effect of chemical functionalization on thermal transport of carbon nanotube composites. Applied Physics Letters, 85, 2229-2231 (2004).

DOI: $\underline{10.1063 / 1.1794370}$

[19] Liu C. H., Fan S. S.: Effects of chemical modifications on the thermal conductivity of carbon nanotube composites. Applied Physics Letters, 86, 123106/1123106/3 (2005).

DOI: $\underline{10.1063 / 1.1887839}$

[20] Yu A., Itkis M. E., Bekyarova E., Haddon R. C.: Effect of single-walled carbon nanotube purity on the thermal conductivity of carbon nanotube-based composites. Applied Physics Letters, 89, 133102/1133102/3 (2006).

DOI: $10.1063 / 1.2357580$
[21] Xu Y., Ray G., Abdel-Magid B.: Thermal behavior of single-walled carbon nanotube polymer-matrix composites. Composites Part A: Applied Science and Manufacturing, 37, 114-121 (2006).

DOI: 10.1016/j.compositesa.2005.04.009

[22] Tsangaris G. M., Psarras G. C., Kouloumbi N.: Electric modulus and interfacial polarization in composite polymeric systems. Journal of Materials Science, 33, 2027-2037 (1998).

DOI: 10.1023/A:1004398514901

[23] Abdul-Jawad S., Alnajjar A., Abdallah M. H.: AC impedance, permittivity and modulus spectroscopy of lead chloride single crystal. Applied Physics A: Materials Science and Processing, 64, 199-201 (1997). DOI: $10.1007 / \mathrm{s} 003390050464$

[24] Yu S., Hing P., Hu X.: Dielectric properties of polystyrene-aluminum-nitride composites. Journal of Applied Physics, 88, 398-404 (2000).

DOI: $10.1063 / 1.373672$

[25] Bobnar V., Levstik A., Huang C., Zhang Q. M.: Enhanced dielectric response in all-organic polyaniline poly(vinylidene fluoride-trifluoroethylenechlorotrifluoroethylene) composite. Journal of NonCrystalline Solids, 353, 205-209 (2007).

DOI: $10.1016 /$ j.jnoncrysol.2006.10.003 